\title{
Validation of respiratory inductive plethysmography using the Qualitative Diagnostic Calibration method in anaesthetized infants
}

\author{
K. Brown*, C. Aun , E. Jackson§, A. Mackersie», D. Hatch», J. Stocks\$
}

Validation of respiratory inductive plethysmography using the Qualitative Diagnostic Calibration method in anaesthetized infants. K. Brown, C. Aun, E. Jackson, A. Mackersie, D. Hatch, J. Stocks. CERS Journals Ltd 1998.

ABSTRACT: The aim of this study was to compare tidal volume $(V T)$ derived from the Qualitative Diagnostic Calibration (QDC) method (VT,QDC) with measurements from pneumotachography (VT,PNT) in anaesthetized infants.

Measurements were made during spontaneous (SV) and intermittent positive pressure (IPPV) ventilation, sighs and airway occlusions. The VT,DIF was the difference between $V \mathrm{~T}, \mathrm{QDC}$ and $V \mathrm{~T}, \mathrm{PNT}(\% V \mathrm{~T})$. The contribution of the ribcage (rc) to $V \mathrm{~T}, \mathrm{QDC}$ (\% rc) and the thoracoabdominal phase lag were also derived. Twenty-eight infants, mean (sD) age 14.0 (6.2) months were studied.

$V T$,QDC represented $V$ T,PNT most closely when Š20 breaths were analysed. There was close agreement during SV immediately after the calibration period $(95 \%$ limits of agreement (LA; QDC - PNT) -2.3, 3.0\%). The 95\% LA increased to -9.6, 10.2\% after $10 \mathrm{~min}$. Accuracy diminished during IPPV (95\% LA -38, 31\%), and sighs. During airway occlusions, when $V$ T,PNT was zero, the $95 \%$ LA were $-6.3,4.1 \mathrm{~mL}^{-\mathrm{kg}^{-1}}$. Mean phase lag was 36 and $2 \%$, respectively, during SV and IPPV $(p<0.05)$. The \% rc appeared to be overestimated, being in excess of $50 \%$ in infants under 12 months.

The Qualitative Diagnostic Calibration method used to estimate tidal volume in anaesthetized infants was limited by the need to analyse $\mathbf{S} 20$ breaths and by a loss of within-subject accuracy if measurement conditions or pattern of breathing changed. Eur Respir J 1998; 12: 935-943.
*Dept of Anaesthesia, Montreal Children's

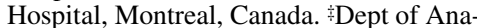
esthesia and Intensive Care, The Chinese University of Hong Kong, Hong Kong. \$Portex Anaesthesia, Intensive Therapy and Respiratory Medicine Unit, Institute of Child Health, 30 Guilford Street, London, UK

Correspondence: K. Brown, 2300 Tupper St, Montreal PQ, H3H 1P3, Canada, Fax: 5149344341

Keywords: Anaesthesia, infants, qualitative diagnostic calibration method, respiratory inductive plethysmography

Received: December 21997

Accepted after revision May 121998

$\mathrm{K}$. Brown was supported by the Canadian Society of Anaesthetists and the Royal College of Physicians and Surgeons of Canada for a fellowship at the Institute of Child Health. C. Aun was on sabbatical leave at the Institute of Child Health. Salary support for J. Stocks and D. Hatch, and purchase of the Somnostar equipment were provided by SIMS-Portex Plc.
During recent years there has been renewed interest in noninvasive methods of assessing respiratory function in infants, since the use of face masks and pneumotachographs (PNT) have both been shown to affect breathing pattern [1]. Respiratory inductive plethysmography (RIP) is a means of measuring breathing movements without any connections at the airway opening, and therefore has numerous potential applications in infants, including prolonged respiratory monitoring in the intensive care unit or postoperatively. RIP uses inductance coils within elasticated bands to measure the respiratory excursion of the ribcage (rc) and abdomen (abd). The respiratory system is assumed to move with two "degrees of freedom", so that changes in inductance are proportional to changes in ribcage and abdominal volume. The weighted sum of the rc and abd is therefore proportional to the tidal volume $(V \mathrm{~T})$. Studies using RIP have increased our understanding of respiratory control and mechanics [2,3], including the fact that infancy is characterized by abdominal breathing [4], and that halothane anaesthesia is associated with a loss of ribcage recruitment [5].

Whereas uncalibrated RIP is a valuable means of assessing both respiratory timing and various qualitative aspects of $\mathrm{rc}$ and abd asynchrony [6-8], calibration is essential if quantitative changes in ventilation are to be assessed. Such calibration, which involves a two-stage process of deter- mining the relative contributions of rc and abd to each breath and then ascertaining a proportionality coefficient in order to scale the weighted sum to actual tidal volume, has, however, proved to be a major challenge when using RIP in infants. Three main methods of calibration have been proposed, as outlined below.

1) The rc and abd signals are compared with simultaneous recordings of $V \mathrm{~T}$ from a PNT, according to the equation:

$$
V \mathrm{~T}=V_{\mathrm{rc}}+V_{\mathrm{abd}}
$$

where $V$ rc is the inductance (voltage) of the rc signal and Vabd is that of the abd signal. Providing $V \mathrm{~T}$ is measured over a large enough number of breaths, a proportionality coefficient $K$ can then be derived using a least squares or graphical solution to the equation. However, since this always requires the simultaneous use of a PNT, the advantages of RIP are at least partially lost.

2) In 1987, Konno and MEAD [9] described the isovolume manoeuvre, as a means of determining the relative contribution of rc and abd without a PNT. This requires the subject to shift gas gently between the rc and abd during a breath hold or airway occlusion, thereby producing paradoxical movements of the two compartments. Since $V T$ does not change, equation (1) can be rewritten as: 


$$
K=-\operatorname{Vabd} / V \mathrm{rc}
$$

This is, however, unsuitable for infants and young children since it requires the subject's cooperation. In an attempt to overcome this problem, various modifications of the isovolume technique have been proposed for use in infants and children, including the use of different patterns of ventilation, due to changes in body posture, sleep state or airway occlusion, to derive the relative contribution of the rc and abd to the tidal volume and thereby assign appropriate calibration coefficients $[4,5,10-13]$. Nevertheless, these methods generally remain too complex for widespread use.

3) In 1989, SACKNER et al. [14] described a computeri-zed method of calibration termed Qualitative Diagnostic Calibration (QDC). This allows calibration to be perform-ed during natural, quiet breathing by taking advantage of spontaneous variations in the relative contributions of the rc and abd to different breaths to solve equation (1). This method is based on the assumption that, if the subject breathes with a constant tidal volume, the variability of rc and abd displacements, as reflected by their standard deviation, can be used to solve for the proportionality constant $K$, such that:

$$
K=-\mathrm{SD}(\operatorname{Vabd}) / \mathrm{SD}\left(V_{\mathrm{rc}}\right)
$$

The QDC method is based on the same assumptions as those outlined by KonNo and MEAD [9], but requires two additional assumptions. The first is that of a constant $V T$, which is approximated by collecting 5 min of tidal breathing and excluding breaths which differ by $>1$ SD from the mean sum $\left(V_{\mathrm{rc}}+V_{\mathrm{abd}}\right)$. The second assumption is that there is intrinsic variability in the relative contribution of the rc and abd to tidal breathing. These additional assumptions allow the derivation of qualitative calibration coefficients from the breath-to-breath variance of the uncalibrated rc and abd signals, using specialized software $[14,15]$. While this method only provides information about the relative contribution of the rc and abd, the simultaneous use of a PNT allows derivation of the volumetric scaling factors, thus allowing derivation of quantitatively calibrated values for the QDC $V$ T if so desired.

Validation of the QDC method has been performed in both awake adults [14], and in sleeping newborns and infants $[15,16]$, but not in anaesthetized subjects, in whom the contribution of the rc and abd compartments during tidal breathing may not be sufficiently variable to allow derivation of accurate calibration factors using QDC [12]. In addition, there is very little information about how many breaths need to be derived from RIP to reflect $V$ T measured simultaneously from a PNT accurately. This is of relevance both in clinical studies, to ensure collection of sufficient data while avoiding undue prolongation of study protocols, and if RIP is to be used to assess the effect of acute interventions on breathing patterns $[6,17]$, wherein relatively few breaths may be available for analysis.

The aims of this study were to: 1) assess the minimum number of breaths derived from RIP required to represent the $V \mathrm{~T}$ recorded simultaneously from a PNT accurately in anaesthetized infants; 2) evaluate the QDC method in anaesthetized infants by comparing VT,PNT and VT,QDC within the same infant under various measurement conditions (namely, spontaneous ventilation, mechanical ventilation, airway occlusions to simulate obstructive apnoeas, and spontaneous sighs); and 3) examine the relative contribution of the rc and abd during both spontaneous and mechanical ventilation in anaesthetised infants.

\section{Materials and methods}

\section{Study subjects}

Patients were recruited from a randomized study comparing the ventilatory effects of sevoflurane and halothane anaesthesia, the results of which have been reported elsewhere [18]. Infants were eligible for recruitment if they were healthy, fasted, had no history of chest wall deformity, cardiovascular or congenital lung disease, and were undergoing elective peripheral limb or urologic surgery. Approval from the Hospital Ethics Committee and informed written parental consent were obtained.

\section{Study design and protocol}

Patients were premedicated with oral atropine (20 $\mu \mathrm{g} \cdot \mathrm{kg}^{-1}$ ) and randomized to receive either halothane or sevoflurane with $66 \%$ nitrous oxide in oxygen for the induction and maintenance of anaesthesia. After induction of anaesthesia, an appropriately sized laryngeal mask was inserted and the anaesthetic vaporizer adjusted to deliver $2.5 \%$ sevoflurane or $1 \%$ halothane, thereby achieving a similar level of anaesthesia in all infants [18-20]. Following induction of anaesthesia, a regional anaesthetic, using either a caudal or brachial plexus block, was performed with bupivacaine $(0.25 \%)$.

The patients were then placed in the supine position before applying the respiratory inductive plethysmography bands (as described below), and inserting the PNT between the laryngeal mask and the anaesthetic T-piece. Data recording then commenced, during which time care was taken not to move the patient. Following the initial 5min period of recording, which was used for the automated QDC calibration (see below), $V$ T from the PNT and RIP were recorded simultaneously during: 1) spontaneous ventilation $\left(\mathrm{SV}_{0}\right)$ immediately following QDC calibration; and 2) mechanical intermittent positive pressure ventilation $\left(\mathrm{IPPV}_{\mathrm{hi}}\right.$ ) approximately $15 \mathrm{~min}$ after the calibration period. In addition, whenever possible, data were collected during: 1) a repeat period of spontaneous ventilation $\left(\mathrm{SV}_{10}\right), 10$ min after the $\mathrm{SV}_{0}$ data and just prior of collection of the IPPV $\mathrm{Vi}_{\mathrm{hi}}$ data; 2) IPPV at a reduced peak airway pressure of $\left.1.1 \mathrm{kPa}\left(11 \mathrm{cmH}_{2} \mathrm{O}\right)\left(\mathrm{IPPV}_{\text {low }}\right) ; 3\right)$ end-expiratory airway occlusions; and 4) spontaneous sighs.

This protocol enabled evaluation of the optimal breath number to use when reporting $V \mathrm{~T}$ derived from QDC of the RIP signals ( $V \mathrm{~T}, \mathrm{QDC})$, reproducibility of $V \mathrm{~T}, \mathrm{QDC}$ over short-time periods, and the influence of changes in breathing patterns on the accuracy of VT,QDC. During IPPV, the lungs were ventilated with a pressure limited, Nuffield Penlon ventilator and a Newton valve using a peak airway pressure of $1.7 \mathrm{kPa}\left(17 \mathrm{cmH}_{2} \mathrm{O}\right)$ and a rate of $21 \mathrm{bpm}$. The decision on whether to use neuromuscular blockade during periods of mechanical ventilation was left to the attending anaesthetist. 


\section{Methods}

Recording equipment. RIP was measured with the SomnoStar PT ${ }^{\mathrm{TM}}$ (NIMS ${ }^{\mathrm{TM}}$, Sensormedics, The Netherlands). Respitrace bands (Respiband Plus, NIMS ${ }^{\mathrm{TM}}$ ) $1.5 \mathrm{~cm}$ in width, were placed at the level of the fourth intercostal interspace and umbilicus for the measurement of rc and abd, respectively [15]. Flow was measured with a heated Hans Rudolph PNT (\#3719, linear range 0-35 L· $\mathrm{min}^{-1}$, Kansas City, MO, USA), attached to a pressure transducer $( \pm 0.2$ kPa, 431 SCXL0040N, Sensym Inc, Minipitas, CA, USA). The airway opening pressure $(P$ ao $)$ was measured with another pressure transducer $( \pm 5.0 \mathrm{kPa}, 511 \mathrm{SCX} 010 \mathrm{~N}$, Sensym Inc) via a side port attached to the laryngeal mask. To perform the airway occlusions, the PNT was connected to an appropriately sized, nonrebreathing valve (Hans Rudolph 2200's) which separated inspiratory and expiratory flow. A pneumatically activated, hand-operated balloontype shutter (Hans Rudolph 9300) was used to produce airway occlusions, which were timed from the real time display of flow. Flow was calibrated using rotameters, and the volume validated with a volumetric $100 \mathrm{~mL}$ syringe (Hans Rudolph) containing the gas mixture used for anaesthesia.

Pressure was calibrated against a water manometer. All calibrations were checked at the start and end of each study. All signals were sampled at $50 \mathrm{~Hz}$, digitized through the SomnoStar PT (NIMS ${ }^{\mathrm{TM}}$ ) and downloaded onto a personal computer for off-line analysis with the software program RespiEvents ${ }^{\mathrm{TM}}$ (version 4.2e, NIMSTM). The PNT flow was digitally integrated to give volume (VPNT).

Data analysis. As described above, the simultaneous use of a PNT allows derivation of the proportionality coefficients (i.e. scaling factors) for $\mathrm{rc}$ and abd, which are then applied to all QDC data subsequently recorded in that subject. The RespiEvents ${ }^{\mathrm{TM}}$ software program derives the quantitative QDC volume signal (VQDC) by assigning a known volume in that subject (obtained from the VPNT), to the voltage signal of the VQDC (which represents the summed channels of the scaled rc and abd signals), in order to establish the voltage to volume relationship. This is achieved by the user isolating a representative segment of the simultaneously acquired signals during spontaneous breathing immediately following the 5-min QDC period. This calibration factor is then automatically applied to all subsequent data from each subject, in order to display the quantitative QDC $V \mathrm{~T}$ (VT,QDC).

Thoracoabdominal asynchrony was assessed from the time-based (scalar) traces of the rc and abd signals, using the "phase relation over the total breath cycle" (PhRTB), hereafter referred to as the "phase lag". This phase lag expresses the percentage discrepancy between the direction of rc and abd movement over the total breath. A value of $0 \%$ indicates that the rc and abd are moving synchronously throughout the breath cycle (equivalent to a $0^{\circ}$ phase lag), whereas a value of $100 \%$ indicates total paradox (equivalent to a $180^{\circ}$ phase lag) [21]. The contribution of the rc to tidal volume (\%rc) was calculated from the calibrated RIP signals. The difference between $V \mathrm{~T}$,QDC and $V$ T,PNT ( $V$ T,DIF) was calculated such that:

$$
V \mathrm{~T}, \mathrm{DIF} \%=\frac{(V \mathrm{~T}, \mathrm{QDC}-V \mathrm{~T}, \mathrm{PNT})}{(V \mathrm{~T}, \mathrm{QDC}+V \mathrm{~T}, \mathrm{PNT}) / 2} \times 100
$$

Influence of breath number. To determine the minimum number of breaths required when reporting $V \mathrm{~T}, \mathrm{QDC}$, we examined the VT,DIF for up to fifty consecutive breaths from each infant during the initial period of spontaneous breathing $\left(\mathrm{SV}_{0}\right)$. For clusters of $\breve{S} 10$ breaths, average values for $V$ T,PNT and $V$ T,QDC were automatically obtained from the RespiEvents breath averaging algorithm, thus allowing calculation of the cumulative VT,DIF over different numbers of breaths. Since this facility was not automatically available for $<10$ breaths, the first 9 consecutive breaths following the calibration period were exported to a spreadsheet for calculation of the average VT,DIF from clusters of 1-9 breaths. Within-breath variability was also calculated for each of these nine breaths individually, to assess the maximum variability that was likely to occur during analysis of single breaths.

Validation of $\mathrm{VT}, Q D C$. The primary validation of the QDC method was based on the $V$ T,DIF obtained from the initial periods of spontaneous and mechanical ventilation. Based on the analysis of optimal breath number (see Results section), a minimum of 20 breaths was used for the calculation of $V$ T,DIF. Patients in whom fewer than 20 breaths were available for either $\mathrm{SV}_{0}$ or IPPV $\mathrm{V}_{\mathrm{hi}}$ data were excluded from further analysis. Ethical constraints restricted undue prolongation of the study protocol, and data collection periods for $\mathrm{SV}_{10}, \mathrm{IPPV}_{\text {low }}$ and occlusion data were therefore time limited. The $V$ T,DIF derived for the $\mathrm{SV}_{10}$ and IPPV $_{\text {low }}$ were obtained from between 12-35 and 11-19 breaths, respectively. Reported values for the occlusion data were determined from six airway occlusions, whereas the $V$ T,DIF for the sigh data were based on analysis of single breaths. Spontaneous sighs were analysed by exporting the data to a spreadsheet to calculate the VT,DIF. During airway occlusions, when VT,PNT was zero, changes in $V \mathrm{~T}$,QDC during the inspiratory effort against the occlusion $(V \mathrm{~T}, \mathrm{QDC}, \mathrm{OCC})$ were calculated, the duration of the occluded inspiration being defined from the $P$ ao trace. The reported value for $V \mathrm{~T}, \mathrm{QDC}, \mathrm{OCC}$ was expressed in $\mathrm{mL} \cdot \mathrm{kg}^{-1}$ body weight.

Statistical methods. The effect of breath number on VT,DIF was assessed by visual inspection of the cumulative and single breath analysis scatter plots. The accuracy of the QDC method was assessed by calculating the $95 \%$ limits of agreement (LA) between $V \mathrm{~T}, \mathrm{QDC}$ and VT,PNT (i.e. $V$ T,DIF), as described by BLAND and AltMAn [22], during $\mathrm{SV}_{0}$ and IPPV $V_{\text {hi }}$. Similar analyses were performed for the $\mathrm{SV}_{10}$, $\mathrm{IPPV}_{\text {low }}$ occlusion and sigh data. Changes in the group mean difference for the phase lag and \%rc between the $\mathrm{SV}_{0}$ and $\mathrm{IPPV}_{\text {hi }}$ data were assessed with paired t-tests and calculation of the $95 \%$ confidence intervals $\left(\mathrm{SV}_{0}-\mathrm{IPPV}_{\mathrm{hi}}\right)$. A pvalue of $<0.05$, and $95 \%$ LA that did not encompass zero [23], were considered statistically significant.

\section{Results}

Parental consent was obtained from 30 of 31 infants eligible for this study. Measurements were obtained in 28 patients. Less than 20 breaths were available during IPPV in five of these children, who were therefore excluded from all but the cumulative breath analysis. Details of the 23 infants in whom both $\mathrm{SV}_{0}$ and $\mathrm{IPPV}_{\mathrm{hi}}$ were available 
Table 1. - Patient characteristics

\begin{tabular}{lc}
\hline $\mathrm{n}$ & 23 \\
Age mean (SD) months & $14.0(6.2)$ \\
Weight mean (SD) kg & $10.5(2.2)$ \\
Sex M:F & $20: 3$ \\
Caudal:brachial plexus blockade & $21: 2$ \\
Neuromuscular blockage Y:N & $7: 16$ \\
\hline
\end{tabular}

M: male; F: female.

are summarized in table 1 . Measurements during $\mathrm{SV}_{10}$, IPPV $_{\text {low }}$, occlusion and sigh data were obtained in 23, 8, 22 and 9 patients, respectively.

\section{QDC calibration period}

During the 5-min period of QDC calibration, the VT,PNT ranged $4.4-7.9 \mathrm{~mL} \cdot \mathrm{kg}^{-1}\left(\operatorname{mean}(\mathrm{SD}) ; 5.9(0.9) \mathrm{mL} \cdot \mathrm{kg}^{-1}\right)$. The mean(SD) intrasubject coefficient of variation in $V$ T,PNT during this period was 9.7(6.3)\% (range 2.7-28.5\%).

\section{Optimal breath number}

Figure 1 shows how quickly (breathwise) the $V$ T,DIF approached zero during $\mathrm{SV}_{0}$ in the 28 patients during the cumulative breath analysis. By 10 breaths, VT,DIF was $<8 \%$ in all but three patients and by 20 breaths $<5 \%$ in all but one, who had a persistently large $V$ T,DIF irrespective of breath number. This infant was therefore considered a QDC failure and excluded from further analysis. Close inspection of this infant's recordings and medical records did not reveal any specific feature that could be associated with this failure. The mean (95\% LA) VT,DIF for the single breath analysis in each infant during $\mathrm{SV}_{0}$ was $0.18 \%$ (-9.2, $9.4 \%$ ) (fig. 2). Only 10/207 breaths that were analysed individually ( 9 breaths in each of 23 infants) had a VT,DIF in excess of $10 \%$.

\section{Validation of the QDC method}

Spontaneous and mechanical ventilation. Details of ventilatory parameters during spontaneous and mechanical

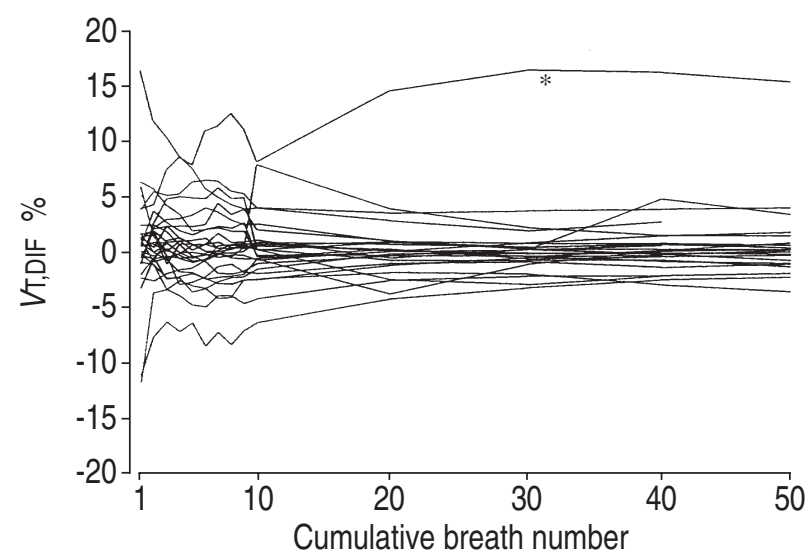

Fig. 1. - Cumulative breath analysis. The difference in tidal volume $(V \mathrm{~T}, \mathrm{DIF})$ (i.e. $((V \mathrm{~T}, \mathrm{QDC}-V \mathrm{~T}, \mathrm{PNT}) /((V \mathrm{~T}, \mathrm{QDC}+V \mathrm{~T}, \mathrm{PNT} / 2)) \times 100$, see Materials and methods section, was calculated from between 1 and 50 breaths in 28 infants during the initial period of spontaneous ventilation. One patient (*) persistently has a $V \mathrm{~T}, \mathrm{DIF}>10 \%$ and was considered a Qualitative Diagnostic Calibration (QDC) method failure. PNT: pneumotachograph.

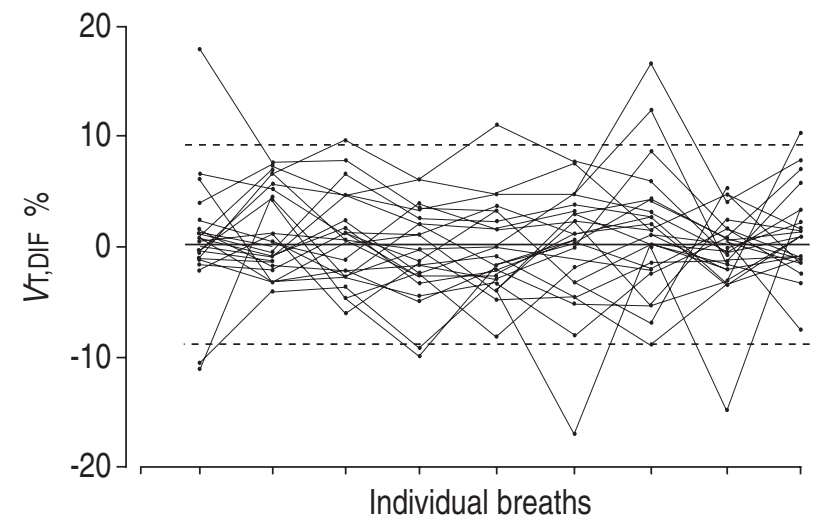

Fig. 2. - Single breath analysis. Individual breath-to-breath variation in tidal volume differences ( $V$ T,DIF; calculated as difference between Qualitative Diagnostic Calibration and pneumotachography tidal volumes), for nine consecutive breaths during an initial period of spontaneous ventilation in the 23 patients with complete sets of data. The mean difference (-) and $95 \%$ limits of agreement (- - -) are indicated

ventilation are summarized in table 2 . The average $V$ T,PNT was $6.1 \mathrm{~mL} \cdot \mathrm{kg}^{-1}$ during the initial period of spontane- ous ventilation $\left(\mathrm{SV}_{0}\right)$, and $6.5 \mathrm{~mL} \cdot \mathrm{kg}^{-1} 10 \mathrm{~min}$ later $\left(\mathrm{SV}_{10}\right)$, increasing during mechanical ventilation to 9.7 and 13.8 $\mathrm{mL} \cdot \mathrm{kg}^{-1}$ for IPPV $\mathrm{low}$ and IPPV $\mathrm{hi}_{\mathrm{h}}$, respectively. Similar group mean values for $V$ T,PNT and $V \mathrm{~T}, \mathrm{QDC}$ were found under all measurement conditions, with no significant differences between the two techniques. However, as reflected by the 95\% LA (table 2), there were marked within-subject differences in some individuals. Inspection of the Bland-Altman plot of the $V$ T,DIF against the average $V$ T (fig. 3) shows that the $95 \%$ LA were narrowest for the $\mathrm{SV}_{0}$ data $(-2.3,3.0 \%)$ and widest for the $\operatorname{IPPV}_{\mathrm{hi}}$ data $(-38.1,31.0 \%)$, with intermediate values for the $\mathrm{SV}_{10}$ data $(-9.6,10.2 \%)$. The VT,DIF for the 8 infants in whom IPPV ${ }_{\text {low }}$ data were obtained, fell within the $95 \%$ limits of agreement of the IPPV $_{\text {hi }}$ data.

Sigh data. The VT,PNT during sighs was $63-160 \%$ greater than that recorded in the same child during spontaneous tidal breathing (table 3). During sighs, VT,DIF (QDC-PNT) ranged $-2.3-29.7 \%$ (fig. 4). In six of the nine (67\%) patients, the sigh $V$ T,DIF exceeded the $95 \%$ LA derived during analysis of single breaths during $\mathrm{SV}_{0}$ (fig. 2).

Occlusion data. During the inspiratory effort against an occluded airway, when $V$ T,PNT was zero, the mean $(95 \%$ LA) VT,QDC,OCC was -1.1 $(-6.3,4.1) \mathrm{mL} \cdot \mathrm{kg}^{-1}$, ranging -7.7$3.6 \mathrm{~mL} \cdot \mathrm{kg}^{-1}$ (fig. 5). This difference represented $-140 \%$ to $72 \%$ of the mean baseline $V \mathrm{~T}, \mathrm{QDC}$ during $\mathrm{SV}_{0}$.

Thoracoabdominal phase lag. The mean thoracoabdominal phase lag was $36 \%$ during both the QDC calibration period and $\mathrm{SV}_{0}$, and $31 \% 10$ min later during $\mathrm{SV}_{10}$ (table 2). There were no spontaneous respiratory efforts during the collection of the IPPV data, as evidenced by a phase lag $<3 \%$ and narrow rc-abd Lissajous loops [21]. The magnitude of the phase lag during the QDC calibration period had no apparent effect on the magnitude of $V$ T,DIF under any measurement condition (data not shown).

Partitioning of tidal volume. The mean (\%rc) contribution to $V \mathrm{~T}$ was similar during $\mathrm{SV}_{0}, \mathrm{IPPV}_{\text {hi }}, \mathrm{SV}_{10}$ and $\mathrm{IPPV}_{\text {low }}$ (table 2). The values during $\mathrm{SV}_{0}$ are plotted against age in 
Table 2. - Details of ventilatory parameters during spontaneous (SV) and intermittent positive pressure (IPPV) ventilation

\begin{tabular}{|c|c|c|c|c|c|c|}
\hline & \multicolumn{2}{|c|}{$\mathrm{SV}_{0}$} & \multicolumn{2}{|c|}{$\mathrm{SV}_{10}$} & $\mathrm{IPPV}_{\mathrm{hi}}$ & $\mathrm{IPPV}_{\text {low }}$ \\
\hline Breaths $n$ & 31.5 & $(3.9)$ & 28.0 & $(6.2)$ & $(2.6)$ & $(3.2)$ \\
\hline \multicolumn{7}{|l|}{ Tidal volume $\mathrm{mL} \cdot \mathrm{kg}^{-1}$} \\
\hline$V$ T,PNT & 6.1 & $(0.9)$ & 6.5 & $(1.0)$ & 13.8 & $(2.1)$ \\
\hline$V \mathrm{~T}, \mathrm{QDC}$ & 6.0 & $(0.9)$ & 6.5 & $(0.9)$ & 13.5 & 10.2 \\
\hline 95\% LA(QDC-PNT) & \multicolumn{2}{|c|}{$-0.24,0.16$} & \multicolumn{2}{|c|}{$-0.59,0.63$} & $-4.8,4.0$ & $-1.8,2.6$ \\
\hline Phase lag \% & 35.9 & (15.9) & 30.9 & (14.2) & $(2.0)$ & (1.6) \\
\hline$\%$ rc $\%$ & 50.2 & (26.5) & 50.0 & $(24.2)$ & $54.3 \quad(10.8)$ & (13.3) \\
\hline
\end{tabular}

Results presented as mean (SD). Weight-corrected tidal volume from the pneumotachograph (VT,PNT) and Qualitative Diagnostic Calibration (VT,QDC) are shown. Phase lag: phase relation of the total breath; \%rc: the percentage ribcage contribution to the tidal breath. $\mathrm{SV}_{0}$ : initial SV; $\mathrm{SV}_{10}: \mathrm{SV}$ after $10 \mathrm{~min}$; $\mathrm{IPPV}_{\text {hi: }}$ IPPV at high peak airway pressure; IPPV $\mathrm{V}_{\text {low }}$ IPPV at low peak airway pressures; LA: limits of agreement.

figure $6 \mathrm{a}$. In infants $<12$ months of age, the change from spontaneous to mechanical ventilation was associated with an unexpected decrease in \%rc during IPPV $_{\mathrm{hi}}$ in all but two infants (fig. 6b), whereas in older children the same change in ventilatory strategy was associated with an increase in \%rc in all but one infant (fig. 6c). In the majority of infants, the \%rc contribution during a sigh was lower than that observed in the same child during tidal breathing.
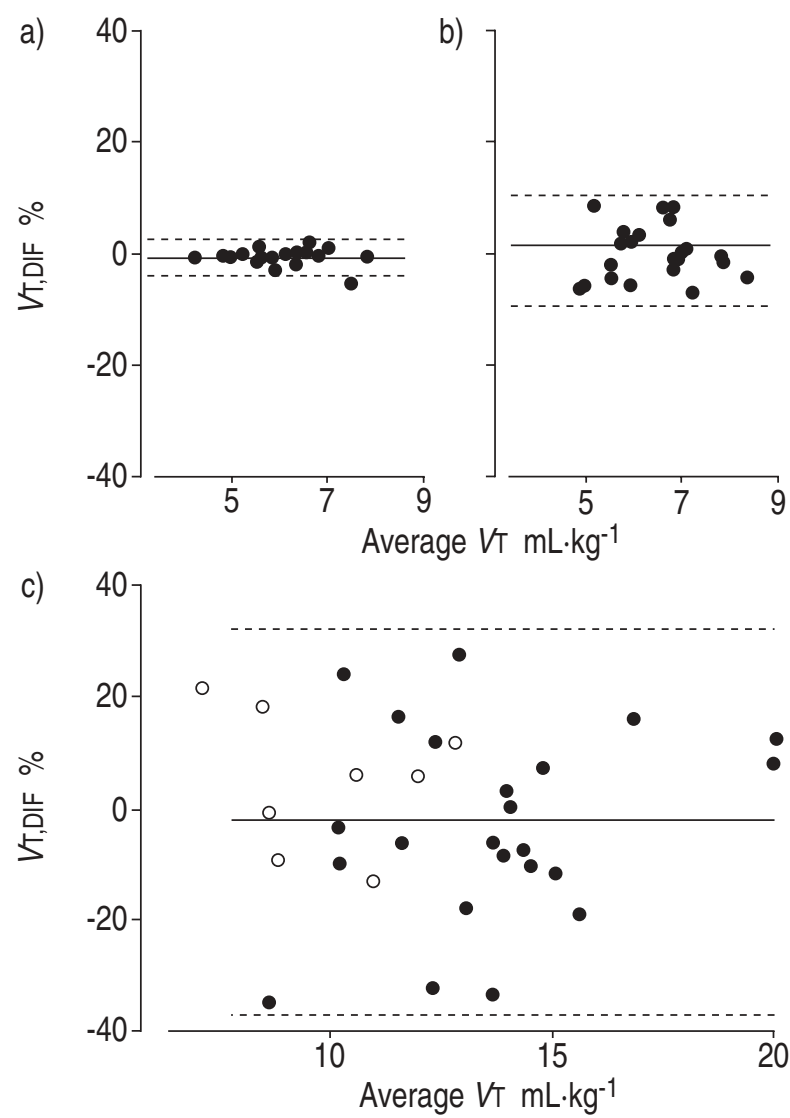

Fig. 3. - Differences between Qualitative Diagnostic Calibration tidal volumes ( $V$ T,QDC) and pneumotachography tidal volumes ( $V$ T,PNT) under various patterns of ventilation. Results are presented using Bland-Altman plots [22] of the difference in tidal volumes (VT,DIF; VT,QDC - VT,PNT), against the average tidal volume $(V \mathrm{~T})$. The mean difference $(-)$ and $95 \%$ limits of agreement (- - -) are indicated. The graphs represent data collected during: a) spontaneous ventilation immediately following an initial period of spontaneous ventilation; b) $10 \mathrm{~min}$ after spontaneous ventilation the calibration period; and c) intermittent positive pressure ventilation with both high ( $\bullet$ ) and low ( o ) peak airway pressures (see text)

\section{Discussion}

This study assessed the QDC method of RIP calibration in 28 anaesthetized infants by comparison with simultaneous recordings from a calibrated PNT, and found that the number of breaths analysed influenced the accuracy with which mean $V$ T,QDC approximated mean $V$ T,PNT. With the exception of one infant, an acceptable agreement between $V$ T,QDC and VT,PNT could be obtained immediately after the calibration period when measurement conditions and breathing patterns resembled those during the calibration period. However, in this population of anaesthetized infants, the calibration coefficients derived by QDC were not sufficiently robust to maintain accuracy when the pattern of breathing changed.

\section{Optimal breath number}

We have previously shown that a limited breath number may not give representative values for respiratory timing parameters, regardless of the method of measurement [24]. In this study, we wanted to determine the minimum number of breaths that should be analysed from RIP in order to reflect simultaneous $V \mathrm{~T}, \mathrm{PNT}$ recordings accurately. The number of breaths used to derive RIP tidal volume in previous reports ranges from $4[14,17]$ to 40 [6] breaths. In this study we found that while the magnitude of $V$ T,DIF increased $<10$ breaths, minimal further accuracy was achieved once breath number exceeded 20 (fig. 1). It

Table 3. - Individual results in nine patients who had spontaneous sighs

\begin{tabular}{lccccc}
\hline $\begin{array}{l}\text { Subject } \\
\text { No. }\end{array}$ & $\begin{array}{c}\text { Increase in } \\
\begin{array}{c}\text { VT,QDC } \\
\text { during sigh } \\
\%\end{array}\end{array}$ & $\begin{array}{c}\text { \%rc } \\
\text { before } \\
\text { sigh }\end{array}$ & $\begin{array}{c}\text { \%rc } \\
\text { during } \\
\text { sigh }\end{array}$ & $\begin{array}{c}\text { \%rc } \\
\text { after } \\
\text { sigh }\end{array}$ & $\begin{array}{c}\text { VT,DIF } \\
\text { (QDC-PNT) } \\
\text { during sigh } \\
\%\end{array}$ \\
\hline 1 & 198 & 88 & 52 & 92 & 30 \\
2 & 170 & 69 & 62 & 72 & 12 \\
3 & 171 & 19 & 12 & 19 & 18 \\
4 & 236 & 80 & 58 & 82 & 9 \\
5 & 257 & 49 & 35 & 40 & -2 \\
6 & 269 & 14 & 14 & 8 & 13 \\
7 & 181 & 44 & 45 & 48 & 2 \\
8 & 234 & 80 & 87 & 85 & 20 \\
9 & 163 & 65 & 40 & 69 & 24
\end{tabular}

The $V$ T,DIF during a sigh is expressed as a per cent of the average tidal volume. VT,QDC: Qualitative Diagnostic Calibration tidal volume; \%rc: percentage ribcage contribution; PNT: pneumotachography. 


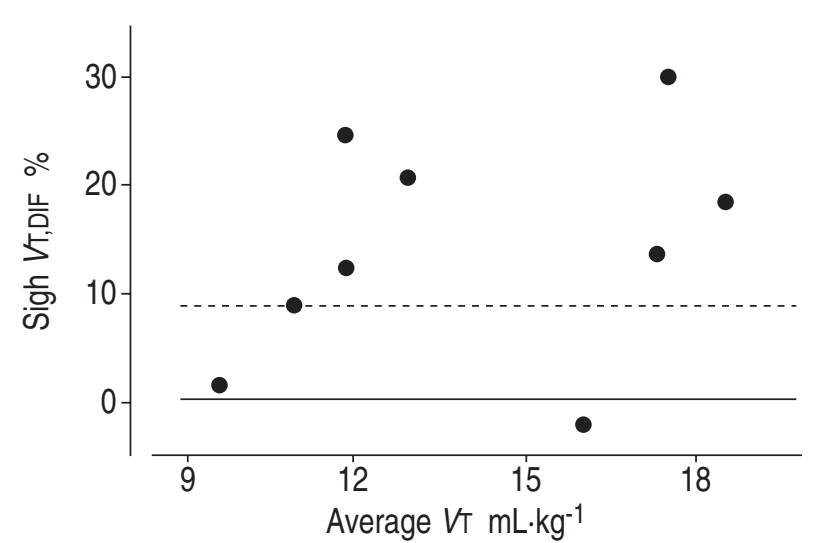

Fig. 4. - Differences between Qualitative Diagnostic Calibration tidal volumes (VT,QDC) and pneumotachography tidal volumes (VT,PNT) in nine patients during spontaneous sighs. The mean difference ( - ) and upper 95\% limit of agreement (- - -) obtained during analysis of single breaths (fig. 2) are superimposed.

thus appears that representative values can be obtained in anaesthetized infants if at least 20 breaths are used. However, more breaths may be required in nonanaesthetized infants, especially if the breathing pattern is irregular.

\section{Validation of QDC method}

Spontaneous ventilation. During spontaneous ventilation, we found excellent agreement between $V$ T,QDC and $V$ T,PNT immediately after the calibration period $\left(\mathrm{SV}_{0}\right)$ (fig. 3), as has been previously reported [14]. Indeed, although the mean value of $V$ T,DIF during $\mathrm{SV}_{0}$ in this study $(-0.7 \%)$ is identical to that previously reported in sleeping infants [16], the 95\% LA in the present study are considerably lower. This may reflect the fact that: 1) sleeping infants have a more variable breathing pattern than anaesthetized infants; 2) we analysed a larger number of breaths than used previously; 3 ) we excluded one patient whose $V$ T,DIF was persistently in excess of $15 \%$ (fig. 1) from further analysis; and/or 4) the $\mathrm{SV}_{0}$ data in the present study were collected immediately after the calibration period. It should be noted that the infants recruited to this study were all "healthy", and that it may not be possible to achieve such

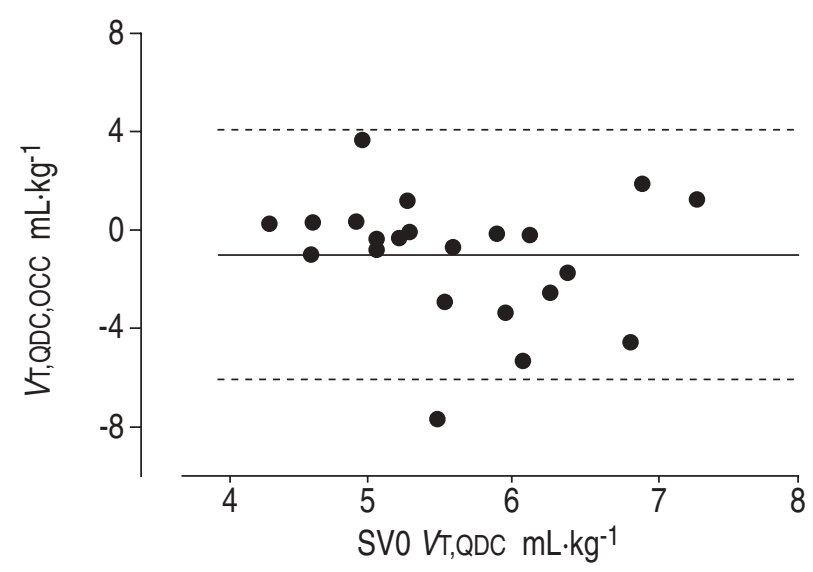

Fig. 5. - Changes in Qualitative Diagnostic Calibration tidal volumes (VT,QDC) during complete airway occlusions (VT,QDC,OCC) in 22 patients. Results are plotted against the weight corrected mean value for $V$ T,QDC in each infant. The group mean $(-)$ and $95 \%$ limits of agreement (- - - ) for $V \mathrm{~T}, \mathrm{QDC}, \mathrm{OCC}$ are also indicated.
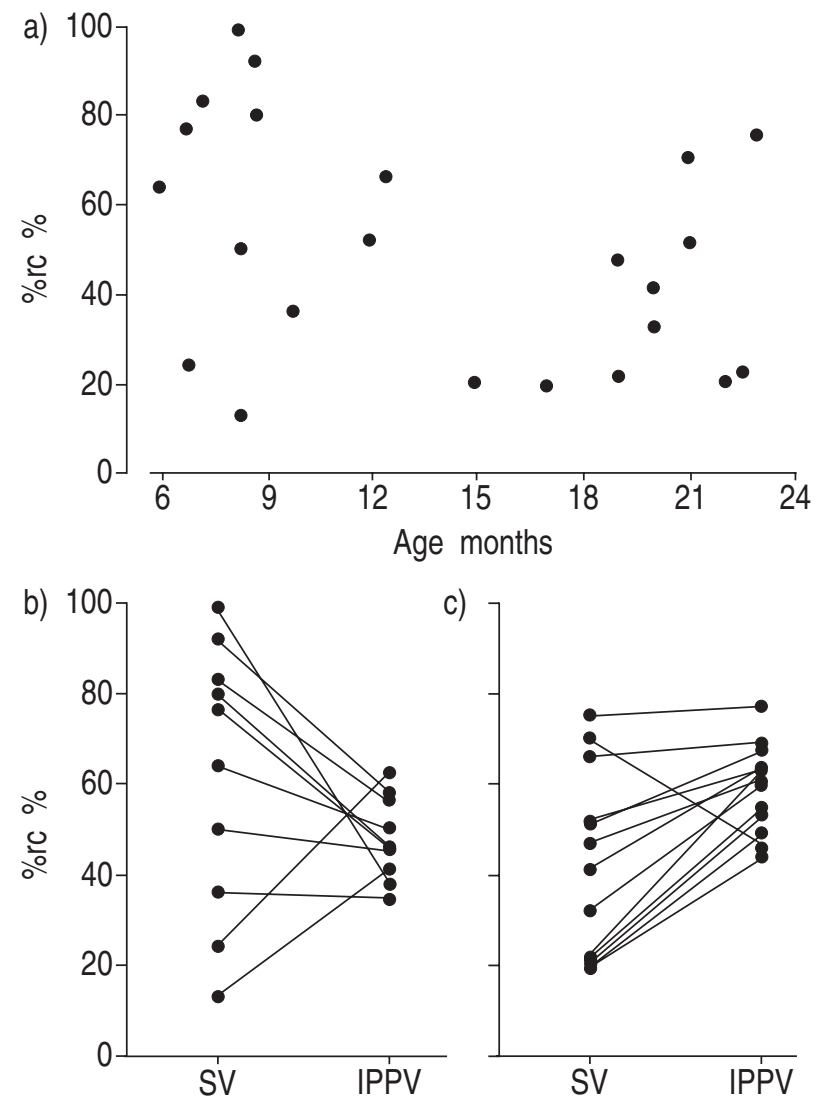

Fig. 6. - The effect of age on the percentage ribcage contribution (\%rc) during spontaneous ventilation $\left(\mathrm{SV}_{0}\right)$. a) A disproportionate number of infants below 12 months of age had a \%rc $>50 \%$ during $\mathrm{SV}_{0}$. b) a change from spontaneous (SV) to intermittent positive pressure (IPPV) ventilation was associated with a decreased \%rc in the majority of infants aged below 12 months. c) the same change in ventilatory strategy increased \%rc in most of the infants above 12 months.

good agreement in sick infants in whom, as discussed below, the extent to which body surface measurements reflect changes at the airway opening may be limited by uneven distribution of ventilation. The limits of agreement between QDC and PNT increased from approximately $\pm 3- \pm 10 \%$ in the 10 min time period between the $\mathrm{SV}_{0}$ and the $\mathrm{SV}_{10}$ data in the current study, suggesting that there may have been some instability of the calibration factors with time, since the breathing pattern over this period remained relatively constant (table 2). Possible reasons for this are discussed below.

Mechanical ventilation. While the group mean VT,DIF remained acceptable during $\operatorname{IPPV}_{\text {hi }}$ (table 2), the 95\% LA for $V$ T,DIF increased dramatically $(-38.1,31.0$, fig. 3). It is unlikely that the magnitude of the VT per se had an important impact on $V$ T,DIF, since similar percentage differences were observed between the IPPV $\mathrm{V}_{\text {hi }}$ and IPPV $\mathrm{V}_{\text {low }}$ data despite different $V$ T (fig. 3). It is also unlikely that a leak around the laryngeal mask contributed to these findings, since: 1) its placement included a check for leaks; 2) there was no evidence of leak on the PNT data [25]; 3) there was no clinically detectable leak during IPPV; and 4) $V$ T,DIF was randomly distributed around zero. The observed discrepancy during IPPV was far more marked than that simply due to a time effect, since these data were collected immediately after the $\mathrm{SV}_{10}$ period. 
Spontaneous sighs. Although VT,QDC generally estimated $V$ T,PNT within $10 \%$ during analysis of single breaths when identical measurement conditions to those during the calibration period were maintained (fig. 2), much larger discrepancies occurred during single breath analysis when breathing pattern changed as, for example, during sighs. Thus, the VT,DIF exceeded $10 \%$ in six, and $20 \%$ in three of the nine patients in whom sighs were performed (fig. 4). This suggests that the QDC technique may not be suitable for tracking acute changes in end expiratory or $V \mathrm{~T}$ in response to interventions such as the application of positive end-expiratory pressure, as has been proposed in the past, since such interventions are invariably associated with marked changes in respiratory pattern and timing.

Airway occlusions. While it is also possible that the pattern of chest and abdominal recruitment during imposed airway obstructions may not reflect those occurring during obstructive apnoeas, the paradoxical movements of rc and abd during the airway occlusions should have resulted in a net zero volume change. However, the $V \mathrm{~T}$,QDC,OCC ex-ceeded $\pm 2 \mathrm{~mL} \cdot \mathrm{kg}^{-1}$ in a number of patients (fig. 5), an error that could not be attributed to a leak, since the $V$ T,PNT approximated zero throughout the occlusion and assumed the same end expiratory baseline postocclusion [25]. It should be noted that although the error was large (fig. 5), it was skewed towards negative values for $V T$,QDC,OCC such that only one child had a value in excess of $50 \%$ of the unoccluded $V \mathrm{~T}$. However, the findings of this study do confirm previous observations that the occlusion technique is unlikely to be a useful method of calibrating RIP in infancy [26].

Potential sources of error. Several factors could have contributed to these observations, including changes in the pattern of breathing and violation of some of the underlying assumptions of the technique. The QDC method is based on several very broad assumptions which may not be valid in the sick or anaesthetized child. Satisfactory calibration during the initial $5 \mathrm{~min}$ recording period is dependent both on a constant $V \mathrm{~T}$, and on variability in the relative contribution of the rc and abd. In an attempt to achieve consistency of $V \mathrm{~T}$, the RespiEvents ${ }^{\mathrm{TM}}$ software program automatically excludes breaths that are without $1 \mathrm{SD}$ of the mean summed signal [14]. However, this algorithm for data exclusion assumes that the distribution of the variability of tidal breathing is normally distributed, and may distort any data which is skewed, a situation that could well occur, especially in young or sick infants. Unfortunately, the RespiEvents ${ }^{\mathrm{TM}}$ software does not allow the user to access the exclusion criteria, or assess the intrinsic variability of the RIP signals during the calibration period. This made it impossible to assess whether the relative contribution of the rc and abd compartments to $V \mathrm{~T}$ was sufficiently variable during the $5 \mathrm{~min}$ of QDC calibration to derive robust coefficients in these anaesthetised infants [12].

The QDC method also assumes that the respiratory system can be represented by a single compartment model, with instantaneous pressure equilibration throughout the system, which may prove to be a significant limitation in infants. The presence of any ventilation inhomogeneities, the magnitude of which will vary according to the pattern of breathing, will also influence the relationship between volume changes measured by RIP and a PNT, irrespective of the method of calibration.

As noted above, there did seem to be some instability of calibration with time, a factor that has been relatively neglected in the literature and which requires further investigation in studies that do not have the time constraints of the current one. While beyond the scope of the present manuscript, a critical evaluation of the mathematics underlying the QDC method, including computer simulations of the potential errors that could arise under circumstances that violate the underlying assumptions, would be extremely valuable.

In the present study, it did appear that changes in the degree of thoracoabdominal asynchrony may have contributed to the increased error seen with changes in ventilatory strategy [3]. While there was no relationship between the degree of phase lag during the calibration period and the magnitude of VT,DIF during IPPV $\mathrm{V}_{\text {hi }}$, which is in keeping with previous reports $[14,16]$, the IPPV, sigh and occlusion data all exhibited very different patterns of chest wall movement from that occurring during $\mathrm{SV}_{0}$. The degree of thoracoabdominal asynchrony during the QDC calibration period and $\mathrm{SV}_{0}$, when $V \mathrm{~T}$, QDC closely approximated $V$ T,PNT, was identical $(36 \%)$, and remained similar 10 min later during $\mathrm{SV}_{10}(31 \%)$. Similar values have been reported previously in healthy anaesthetized infants [27], and reflect the loss of intercostal muscle tone during halothane anaesthesia. By contrast, the simulated obstructed apnoea produced a completely different pattern of rc and abd recruitment, while the change in the ventilatory strategy from spontaneous to mechanical ventilation was associated with a marked diminution of the phase lag to $<3 \%$. The latter condition was associated with a ten-fold increase in the limits of agreement for VT,DIF compared with $\mathrm{SV}_{0}$. It is well recognized that induction of anaesthesia is associated with marked changes in ventilation and thoracoabdominal asynchrony [27], and that changes in ventilatory strategy from spontaneous to mechanical ventilation during anaesthesia are associated with alterations in ventilation distribution in anaesthetized subjects [28], both of which may have contributed to the discrepancies observed in this study.

Partitioning of tidal volume. The increased error in VT,QDC associated with the different measurement conditions suggests that the derived calibration factors were inaccurate in this study. Furthermore, during a sigh, a breathing pattern usually characterized by a recruitment of the rc [29], the \%rc actually decreased in eight of the nine infants in this study. These concerns were highlighted by the observation that the \%rc was unexpectedly high in the majority of infants, and exceeded $50 \%$ in seven of 10 infants $<12$ months of age (fig. 6a). This high ribcage contribution to $V \mathrm{~T}$ is not consistent with studies showing an inhibition of the intercostal musculature [30] and derecruitment of the rc during halothane anaesthesia [5], and is also in contrast to values reported from sleeping infants $<9$ months of age, in whom \%rc was $<50 \%$ when assessed with RIP calibrated with a nonQDC method [4]. Furthermore, amongst infants $<12$ months, mechanical ventilation decreased the \%rc contribution in all but the two who had the lowest \%rc $(<30 \%)$ during $\mathrm{SV}_{0}$ (fig. 6b). In contrast, IPPV was associated with an increased \%rc, in all but one of the older infants, which is consistent with a redistribution of 
$V$ T to the thoracic compartment during passive inflation of the lungs (fig. 6c). It should be noted that the \%rc was initially $<50 \%$ during spontaneous ventilation in 9/11 infants $>12$ months of age (fig. 6a), observations more in keeping with the published literature [4].

Thoracoabdominal asynchrony may have contributed to erroneous estimations of \%rc, since partitioning of $V \mathrm{~T}$ into the relative contribution of rc and abd may be invalid during chest wall distortion, especially if the deformation of the rc introduces a phase lag between transverse and anteroposterior diameters [3]. In addition, recruitment of the abdominal expiratory muscles during anaesthesia [31], and simulated obstructive apnoea may have decreased the compliance of the abdominal wall, thereby decreasing the apparent contribution of the abd compartment to the $V \mathrm{~T}$ during certain measurement conditions. However, since there was no relationship between the degree of phase lag and age in the current study this would not explain the age-related discrepancies we found.

Infants may present an additional challenge for the calibration of RIP, namely the optimal placement of the Respitrace bands. The excursion of the infant's diaphragm results in both anterior displacement of the abdominal wall and lateral displacement of the lower ribcage [32]. Placement of the abdominal band at or below the level of the umbilicus, as recommended [15], may underestimate this latter action and hence underestimate the contribution of the abd (and thereby overestimate that of the ribcage) to the tidal breath. In contrast, the increased excursions associated with the larger $V$ T during IPPV and sighs may well have been reflected more accurately, resulting in an apparent increase in the amplitude of the abd signal, and hence a relative decrease in that of the $\mathrm{rc}$. Indeed, the decrease in \%rc in many infants, during both mechanical ventilation (fig. 6b) and a spontaneous sigh, supports the notion of an underestimate of the diaphragmatic excursion by the abd signal during tidal breathing. The recommended Respitrace bands for use with the SomnoStar PT ${ }^{\mathrm{TM}}$ are only 1.5 $\mathrm{cm}$ in width. This narrow band, placed at the umbilicus, was probably a major contributor to the systematic underestimate of the contribution of the abd compartment to $V \mathrm{~T}$ in infants during spontaneous ventilation. Evaluation of optimal band width and position when using RIP in infants requires urgent attention.

\section{Conclusions}

In this study, we found that the use of the Qualitative Diagnostic Calibration method as a noninvasive means of obtaining quantitatively accurate estimates of tidal breathing in anaesthetized infants was limited by the need to analyse Š20 breaths, and by a loss of within-subject accuracy if measurement conditions or pattern of breathing changed. Further work is required to determine whether similar errors occur in nonanaesthetized infants, and to investigate the unexpectedly high percentage ribcage contribution values found in this study.

Acknowledgements: The authors would like to thank M. Henschen, A-F. Hoo, and I. Dundas for their support and help in ordering and setting up the recording equipment and $\mathrm{J}$. Veness for biomedical engineering support. Gratitude is extended to the departments of Orthopaedic, Plastic and Urologic Surgery and the Theatre and Ward staff at Great Ormond Street Hospital for Children NHS Trust for their support of this study.

\section{References}

1. Dolfin T, Duffty P, Wilkes D, England S, Bryan H. Effects of a face mask and pneumotachograph on breathing in sleeping infants. Am Rev Respir Dis 1983; 123: 977-979.

2. Pengelly LD, Tarshis AM, Rebuck AS. Contribution of ribcage and abdomen-diaphragm to tidal volume during $\mathrm{CO}_{2}$ rebreathing. J Appl Physiol 1979; 46: 709-715.

3. Yan S, Sliwinski P, Macklem PT. Association of chest wall motion and tidal volume responses during $\mathrm{CO}_{2}$ rebreathing. J Appl Physiol 1996; 81: 1528-1534.

4. Hershenson MB, Colin AA, Wohl MEB, Stark AR. Changes in the contribution of the ribcage to tidal breathing during infancy. Am Rev Respir Dis 1990; 141: 922-925.

5. Tusiewicz K, Bryan AC, Froese AB. Contributions of changing ribcage-diaphragm interactions to the ventilatory depression of halothane anaesthesia. Anesthesiology 1977; 47: 327-337.

6. Springer C, Godfrey S, Vilozni D, Bar-Yishay E, Noviski $\mathrm{N}$, Avital A. Comparison of respiratory inductance plethysmography with thoracoabdominal compression in bronchial challenges in infants and young children. Am J Respir Crit Care Med 1996; 154: 665-669.

7. Diaz CE, Deoras KS, Allen JL. Chest wall motion before and during mechanical ventilation in children with neuromuscular disease. Pediatr Pulmonol 1993; 16: 89-95.

8. Gaultier C, Praud JP, Canet E, Delaperche MF, D'Allest AM. Paradoxical inward ribcage motion during rapid eye movement sleep in infants and young children. $J$ Dev Physiol 1987; 9: 391-397.

9. Konno K, Mead J. Measurement of the separate volume changes of ribcage and abdomen during breathing. J Appl Physiol 1967; 22: 407-422.

10. Dolfin T, Duffty P, Wilkes DL, Bryan MH. Calibration of respiratory induction plethysmography (Respitrace ${ }^{\circledR}$ ) in infants. Am Rev Respir Dis 1982; 126: 577-579.

11. Adams JA, Zabaleta IA, Sackner MA. Comparison of supine and prone noninvasive measurements of breathing patterns in full-term newborns. Pediatr Pulmonol 1994; 18: 8-12.

12. Sartene R, Dartus C, Bernard JL, Mathieu M, Goldman MD. Comparison of thoracoabdominal calibration methods in normal human subjects. J Appl Physiol 1993; 75: 2142-2150.

13. Revow MD, England SJ, Stogryn HAF, Wilkes DL. Comparison of calibration methods for respiratory inductive plethysmography in infants. J Appl Physiol 1987; 63: 1853-1861.

14. Sackner MA, Watson H, Belsito AS, et al. Calibration of respiratory inductive plethysmograph during natural breathing. J Appl Physiol 1989; 66: 410-420.

15. Adams JA. Respiratory inductive plethysmography. In: Stocks J, Sly PD, Tepper RS, Morgan WJ, eds. Infant Respiratory Function Testing. New York, John Wiley, 1996; pp. 139-164.

16. Adams JA, Zabaleta IA, Stroh D, Johnson P, Sackner MA. Tidal volume measurements in newborns using respiratory inductive plethysmography. Am Rev Respir Dis 1993; 148: 585-588.

17. Rusconi F, Gagliardi L, Aston H, Silverman M. Respiratory inductive plethysmography in the evaluation of lower airway obstruction during methacholine challenge in infants. Pediatr Pulmonol 1995; 20: 396-402.

18. Brown K, Aun C, Stocks J, Jackson E, Mackersie A, Hatch DJ. A comparison of the respiratory effects of sevoflurane and halothane anaesthesia in infants and young children. Anesthesiology 1998; 89: 86-92. 
19. Lerman J, Sikich N, Kleinman S, Yentis S. The pharmacology of sevoflurane in infants and children. Anesthesiology 1994; 80: 814-824.

20. Gregory GA, Eger EI, Munsen ES. The relationship between age and halothane requirements in humans. Anesthesiology 1969; 30: 488-491.

21. Allen JL, Sivan Y. Measurements of chest wall function. In: Stocks J, Sly PD, Tepper RS, Morgan WJ, eds. Infant Respiratory Function Testing. New York, John Wiley, 1996; pp. 329-355.

22. Bland JM, Altman DJ. Statistical methods for assessing agreement between two methods of clinical measurement. Lancet 1986; i: 307-310.

23. Gardner MJ, Altman DG. Confidence intervals rather than p-values: estimation rather than hypothesis testing. Br Med J 1986; 292: 746-750.

24. Stocks J, Dezateux CA, Jackson EA, Hoo A, Costeloe KL, Wade AM. Analysis of tidal breathing parameters in infancy: how variable is $t \mathrm{PTEF}: t \mathrm{E}$ ? Am J Respir Crit Care Med 1994; 150: 1347-1354.

25. Stocks J, Nothen U, Sutherland P, Hatch DJ, Helms P. Improved accuracy of the occlusion technique for assessing total respiratory compliance in infants. Pediatr Pulmonol 1987; 3: 71-77.

26. Gagliardi L, Rusconi F, Aston H, Silverman M. Occlusion maneuver to detect the relative contribution of ribcage and abdomen to tidal volume using respiratory inductive plethysmography in infants. Pediatr Pulmonol 1996; 21: 132-137.

27. Benameur M, Goldman MD, Ecoffey C, Gaultier C. Ventilation and thoracoabdominal asynchrony during halothane anesthesia in infants. J Appl Physiol 1993; 74: 1591-1596.

28. Froese AB, Bryan AC. Effects of anesthesia and paralysis on diaphragmatic mechanics in man. Anesthesiology 1974; 41: 242-255.

29. Mathew OP, Pronske ML, Clark ML. Relative contribution of ribcage and abdomen during augmented breaths in infants. Pediatr Pulmonol 1988; 4: 134-138.

30. Ochiai R, Guthrie RD, Motoyama EK. Effects of varying concentrations of halothane on the activity of the genioglossus, intercostals, and diaphragm in cats: an electromyographic study. Anesthesiology 1989; 70: 812-816.

31. Shulman DL, Volgyesi GA, Burrows FA, Lerman J, England SJ. Expiratory muscle activity in anesthetized children: effect on the single breath technique. Pediatr Pulmonol 1989; 7: 82-88.

32. Hershenson MB. The respiratory muscles and chest wall. In: Beckerman RC, Brouillette RT, Hunt CE, eds. Respiratory Control Disorders in Infants and Children. Baltimore, MD, Williams \& Wilkins, 1992; pp. 28-46. 